\title{
HIM-SIMS: Correlative SE/Chemical Imaging at the Limits of Resolution.
}

\author{
D. Dowsett, T. Wirtz and L. Yedra
}

Advanced Instrumentation for Ion Nano-Analytics (AINA), MRT Department, Luxembourg Institute of Science and Technology (LIST), 41 rue du Brill, L-4422 Belvaux, Luxembourg

Techniques for nano-metrology and nano-analyisis are crucial for the ongoing investigation of nanoscale processes in disciplines from materials to life sciences. The Helium Ion Microscope (HIM) has become an ideal tool for imaging and nano-patterning [1]. Imaging with helium and neon ions leads respectively to resolutions of $0.5 \mathrm{~nm}$ and $\sim 2 \mathrm{~nm}$ for SE based imaging, while structures with sub $20 \mathrm{~nm}$ feature sizes may be patterned using Ne. Despite these advantages, the analysis capability of the instrument is currently limited. At beam energies of $35 \mathrm{keV}$ helium ions do not lead to the emission of characteristic $\mathrm{X}$-rays from a sample. While some compositional information can be obtained from back scattered helium [2], identifying elemental information is more difficult due to the multiple collisions that occur at energies below $100 \mathrm{keV}$ [3].

In this paper we present the latest results from a prototype Secondary Ion Mass Spectrometry (SIMS) system specifically designed for the Zeiss ORION NanoFab HIM [4-6]. As the probe size of the HIM is below the lateral information limit in SIMS, the lateral resolution is only limited by the probe-sample interactions and not (as in commercial SIMS instruments) the probe size. Further, the in-situ combination of sub-nanometer resolution SE images with ultra-high resolution SIMS images, produces a step change in the information obtained from a single sample analysis: While secondary electron imaging on the HIM yields topographical information on the nanometre and even sub nanometer scale, SE imaging alone is not enough to obtain a deep understanding of the sample. SIMS imaging provides an elemental mapping capability, however as the yield of secondary ions is much lower than the one of electrons and the emission area is much larger, the resolution obtainable is limited to a few tens of nanometers. SIMS images often produce hot spots of a few pixels in size that are statistically significant due to the low background of SIMS ( $<1 \mathrm{cps})$ but yield little information about the size and morphology of the underlying structures that give rise to the signal. By combining both types of information, features too small to be adequately resolved in SIMS may be investigated by SE imaging and the two images sets co-registered and overlayed or fused [7,8]. By combining these two techniques in-situ, artefacts due to contamination or sample modification during transfer between instruments may be avoided. The speed at which it is possible to switch between SIMS and SE imaging in our system (the fastest switch can be made in as little as a few seconds) opens the possibility for entirely new correlative workflows to be developed. Figure 1 shows a correlative study of a lithium titanate and magnesium oxide nanoparticle mixture. Not only do the SIMS maps present the highest resolution SIMS data known to the authors (75-25\% edge resolutions of just $10 \mathrm{~nm}$ have been obtained), the correlation of SIMS images with SE images allows individual nanoparticle clusters to be unambiguously identified. As SIMS is capable of detecting all elements and all isotopes, this in-situ combination offers significant advantages over SEM-EDS.

We will present the status of the prototype development and the latest results in the field of materials science. 


\section{References:}

[1] L. Scipioni et al, J. Vac. Sci. Technol. B 27 (2009) 3250

[2] G. Hlawacek, V. Veligura, R. Van Gastel, B. Poelsema, J. Vac. Sci. Technol. B 32 (2014) 020801

[3] N. Klingner, R. Heller, G. Hlawacek, J. von Borany, J. Notte, J. Uang, S. Facsko, Ultramicroscopy 162 (2016) 91-97

[4] T. Wirtz, N. Vanhove, L. Pillatsch, D. Dowsett, S. Sijbrandij, J. Notte, Appl. Phys. Lett. 101 (4) (2012) 041601-1-041601-5

[5] L. Pillatsch, N. Vanhove, D. Dowsett, S. Sijbrandij, J. Notte, T. Wirtz, Appl. Surf. Sci. 282 (2013) 908-913

[6] T. Wirtz, D. Dowsett, P. Philipp, Helium Ion Microscopy, edited by G. Hlawacek, A. Gölzhäuser, Springer, 2016

[7] P. Gratia, G. Grancini, J.-N. Audinot, X. Jeanbourquin, E. Mosconi, I. Zimmermann, D. Dowsett, Y. Lee, M.l Grätzel, F. De Angelis, K.Sivula, T. Wirtz, M. K. Nazeeruddin, J. Am. Chem. Soc. 138 (49) (2016) 15821-15824

[8] T. Wirtz, P. Philipp, J.-N. Audinot, D. Dowsett, S. Eswara, Nanotechnology 26 (2015) 434001

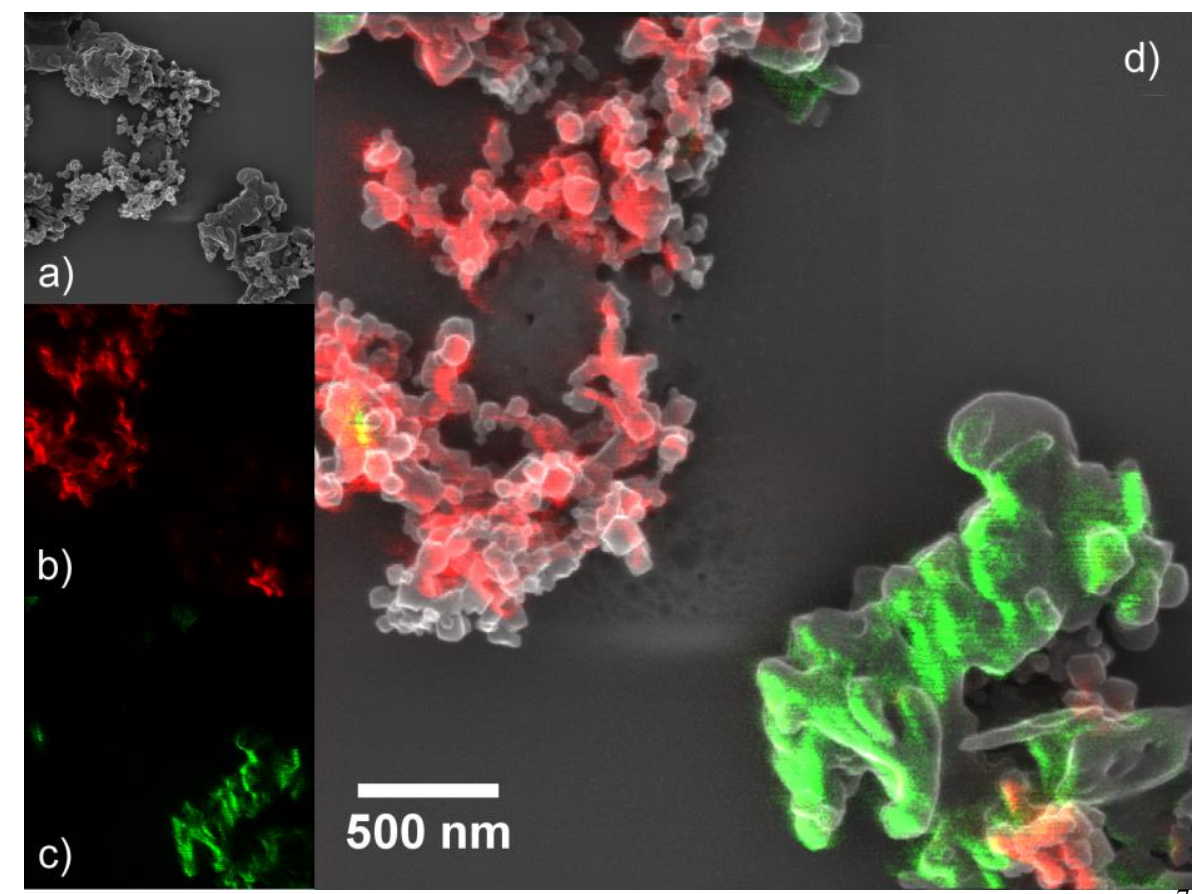

Figure 1. Correlative analysis of LTO/MgO nanoparticle mixture. a) SE image b) ${ }^{7} \mathrm{Li}$ distribution c)

${ }^{24} \mathrm{Mg}$ distribution d) Co-registered SE/SIMS images. The in-situ combination of SE and SIMS allows individual nanoparticle clusters to be identified. 